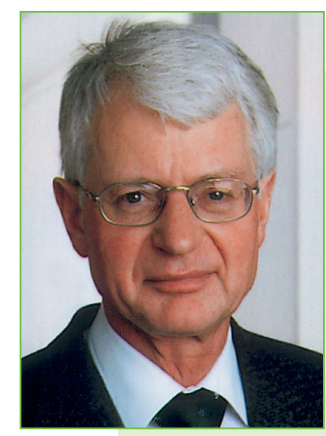

\title{
Phytopharmaka und Phytotherapie 2005: Grund zum Optimismus
}

Vom 8. bis 10. Oktober 2005 fand zum 5. Mal in Berlin der Kongress »Phytopharmaka und Phytotherapie - Forschung und Praxis» statt. Der Autor dieses Editorials hat die wissenschaftlichen Programme bei allen fünf Tagungen mitgestaltet. Die Vorträge und Poster ergeben im Rückblick das folgende Bild: Im Jahr 2000 wurden 29, im Jahr 2001 nur noch 23 Beiträge registriert. Danach wuchs die Zahl der Anmeldungen kontinuierlich, nämlich in 2002 auf 68, in 2004 auf 82 und in diesem Jahr auf 95 Titel. Wer die Tagungen nacheinander besucht hat, wird bestätigen können, dass die wachsende Masse keineswegs zulasten der "Klasse» der Referate gegangen ist. In diesem Jahr konnten von drei beantragten Vorträgen weniger als zwei als solche berücksichtigt werden, was der wissenschaftlichen Qualität weiter zugute kam.

Gewiss ist im berichteten Zeitraum auch die Zahl der beteiligten Fachverbände gewachsen. War es im Jahr 2000 noch die Gesellschaft für Klinische Pharmakologie und Therapie (GKPharm) allein, sind danach sukzessive als Träger der Veranstaltung hinzugekommen: 2001 die Gesellschaft für Phytotherapie (GPT), 2004 die Gesellschaft für Arzneipflanzenforschung (GA) und in diesem Jahr das Komitee Forschung Naturmedizin (KFN). In Bezug auf den inhaltlichen Schwerpunkt dieser Kongresse, fokussiert auf die angewandte Phytotherapie, zehren jedoch alle diese Verbände von denselben Quellen. Der Zuwachs an Beiträgen kann deshalb kaum mit dem Zutritt weiterer Veranstalter erklärt werden.

In diesem Sinne stimmt die Zunahme aktiver Beiträge in 2005 gegenüber 2004 ganz besonders optimistisch. Lagen bzw. liegen doch in zeitlicher Folge vor bzw. nach diesem Kongress zwei Ereignisse, aufgrund derer Pessimisten bereits den jähen Absturz insbesondere der klinischen Forschung mit Phytopharmaka prophezeit hatten: Am 1. April 2004 trat in Deutschland die weitgehende Einschränkung der Erstattungsfähigkeit von pflanzlichen Arzneimitteln durch die GKV in Kraft und am 6. September 2005 wurde mit der 14. AMG-Novelle die europäische Regelung zur "traditionellen« Registrierung von Phytopharmaka in deutsches Recht umgesetzt. Letzteres bedeutet, dass die Zulassung solcher Präparate auch ohne eigenen Forschungsaufwand zur Wirksamkeit möglich sein wird.

Wer allerdings meint, dass mit solchen administrativen Eingriffen die anspruchsvolle Forschung mit Phytopharmaka stehen oder fallen müsste, der irrt. Es sind nämlich nicht allein die Fachkreise in $\mathrm{Zu}$ lassungsbehörden, Arztpraxen und Apotheken, die von modernen pflanzlichen Arzneimitteln mehr als nur ehrwürdige Traditionen fordern. Der mündige Patient selbst wählt zum eigenen Gebrauch bevorzugt diejenigen pflanzlichen Arzneien aus, deren Qualität aktuell und auf dem Boden der Wissenschaft unserer Zeit untermauert wird. Eine solche Triebfeder für die Forschung, die direkt vom Verbraucher kommt, verliert ihre Spannkraft auch nicht durch temporäre regulatorische Ereignisse.

Volker Schulz, Berlin 